\title{
Dental Myths and Taboos: Hurdles to Oral Health
}

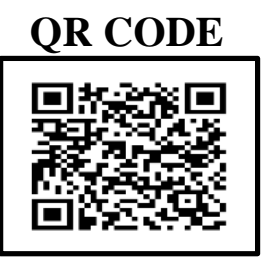

\section{JITENDER DAGAR*1, ARUN RAHAR², NIVEDITA GAUTAM³ ${ }^{3}$ MUNISH DHEERAJ4}

For generations, the dental profession has encountered countless subjects coming up with diverse myths and other illogical beliefs. Most of these have their roots deeply seated in cultural and religious factors. These myths have got a significant impact on oral health of the population, leading to a poor oral health status and ultimately degrading the oral health related quality of life. There is an urgent need to break this chain of unreasoned concepts that is being passed from one generation to the next. It is a difficult task but not impossible. The requirement of the hour is to impart dental health education at grass root level for behavioural modification and dissolving the irrational beliefs for increasing oral health awareness and dental care utilization rate.

KEYWORDS: Dental Myths, Dental Taboos, Beliefs

\section{INTRODUCTION}

There is a social perspective of health from which it cannot be separated and these social factors deeply influence health. Once upon a time, ages ago, the ancient man attributed disease to be a consequence of invasion of human body by evil spirits caused due to the wrath of Gods. ${ }^{1}$ The supernatural theory of disease was advocated on this planet for countless years. Decades ago dental treatment was provided by unqualified dentists which certainly was not based on any scientific logic or dental knowledge and along with this kind of assumption based treatment, taboos developed which became imprinted in minds of people. Taboos are a component of the culture, also leading to a unity among people from a society. Taboos can be defined as subjects, actions, activities or even words that are implicitly prohibited due to social or religious customs.

Myths are referred to as beliefs derived from narratives or stories, mostly of an unknown origin, which strongly influence the life of individuals and their way of living, also impacting their attitude and behaviour towards seeking treatment. People from different religions, cultures, communities and social backgrounds have their own ideologies, misconceptions, beliefs and practices in context to oral diseases and treatments which are inherited and passed from generation to generation.

In a country like India, with major population residing in rural areas, there is a wide diversity of people with different ethnicities, religions, communities, castes and socio-economic backgrounds. This diversity is directly proportional to myths, taboos, misconceptions and false beliefs. Communities with poor accessibility to oral health care delivery systems are more prone to oral diseases. Instead of visiting qualified dental professionals, people show more faith in quacks, and even try to go for spiritual treatment and alternative forms of medicine. $^{2}$ Traditional beliefs and taboos are negatively related with preventive dental health behaviour of population. ${ }^{3}$ There is a marked ignorance and lack of scientific dental awareness among common people in India, mainly for those residing in rural areas and those with less education and dental awareness. A developing country like India is facing countless challenges in rendering oral health needs. Some myths and taboos are a part of every individual. However, these irrelevant but hardly imprinted elements could result in dangerous consequences if followed.

Following are some common misunderstandings related to dentistry that are prevalent in the population-

1. Extraction of teeth, especially upper impairs and adversely affects the eye sight and vision. There is no such detailed fact in literature. Sometimes, extraction becomes mandatory but it has nothing to do with eyesight.

2. There is no need to visit dentist for treating milk teeth as they need not be cared for because they don't last long and these will ultimately be replaced by permanent teeth. Early loss of primary teeth will lead to difficulty chewing and will affect 
the child's nutritional intake. It might also result in drifting of the adjacent teeth causing the permanent teeth to erupt in irregular position ultimately resulting in crowding. ${ }^{4}$

3. Spacing between teeth is a sign of good luck. Spacing has nothing to do with destiny. It results in social stigma affecting the oral health related quality of life.

4. Swelling caused by painful tooth should be fomented with hot water. On the contrary, this procedure increases swelling.

5. Cleaning teeth with fingers is better than cleaning with a toothbrush. Toothbrush bristles can easily intervene the interdental areas and other regions of dentition and oral cavity which cannot be reached by fingers.

6. Abrasives like charcoal, salt and tobacco powder are better than toothpaste for cleaning teeth. These kind of materials abrade the tooth surface leading to sensitivity and even fractures.

7. White teeth are stronger and harder brushing can help achieving it. Every person has got a different shade of teeth mainly depending on the mineral and organic content.

8. It is not good to get dental treatment during pregnancy. Second trimester is safe for a few dental procedures.

9. Old and male dentists are more expert than younger ones and their female counterparts. Expertise does not depend on age and gender.

10. Oral cancer is related to past sins. Oral cancer is multifactorial with genetics, tobacco use, sharp tooth edge, spices and many more.

A common myth among general population is that the teeth become loose after professional cleaning by a dentist. In a study conducted by Farooq $\mathrm{H}$ et al, some of the study subjects reported to believe that scaling will loosen teeth and many of them thought that scaling will increase gaps between teeth. ${ }^{5}$

Keeping tobacco beside a painful tooth reduces tooth pain. This habit results in the chances of oral cancer.

Understanding that myths and taboos about oral diseases are illogical and groundless is important for provision of adequate dental care and health education. As the high prevalence of these myths. ${ }^{1}$

These prevalent myths in a population are mainly backed by a variety of reasons like poor education, religious beliefs and socio-cultural misconceptions and are proportional with poor health seeking behaviour and treatment compliance. These concepts prevent the affected population from obtaining optimum dental care even if they have easy access to it. This kind of false education is deep seated in the society and their understanding by health professionals is essential in order to provide a good care. Stress should be laid down on creating awareness regarding myths about oral health at the individual as well as community level. Oral health education should be provided to all age groups and people from all backgrounds, considering population residing in rural regions more, which could help in raising inner perceptions as such strengthening would furthermore modify unfortunate conduct and practices.

\section{CONCLUSION}

Myths, taboos, misbeliefs and misconceptions become a part of the blood when taken too seriously and inculcated in heart and mind. The social doctrines are mainly because of absence of awareness and lack of education but they are a big hindrance in the way of utilization of dental healthcare services and the road to perfect oral health. There is a need to eliminate and reform unproven and unjustifiable beliefs regarding dental health in order to improve the oral and overall health of the countless misguided countrymen. Importance should be given to oral health education regarding the commonly prevalent myths. There is a need to ascertain the current prevalence of these cultural taboos and beliefs regarding dentistry among the Indian population and to assess the impact of various factors associated with them. The government, dental professionals, dental organisations and Non-Governmental Organisations should come up and work together efficiently in a coordinated way to help in shaping the future oral health programs and creating dental awareness to eradicate these taboos and beliefs which are to some extent fading with the gradual development of education, but are still persisting and are commonly encountered.

\section{REFERENCES}

1. Ahmed B, Imran MF, Amin M, Saeed F. Sources of information and oral hygiene practice among patients visiting dental section of university medical \& dental college, Faisalabad. JUMDC 2010; 1(2):8-10.

2. Singh SV, Tripathi A. A study on prosthodontic awareness and needs of an ageing Indian rural population. J Ind Prosthodont Soc 2007;7:21-3. 
3. Lim LP, Schwarz E, Lo ECM .Chinese health beliefs and oral health practices among the middle-aged and the elderly in Hong Kong. Community Dent Oral Epidemiol 1994;22(5):364-8.
4. Alexander RE. Eleven myths of dentoalveolar surgery. J Am Dent Assoc 1998;129:1271-9.

5. Farooq H, Bukhari SH, Riaz M. Myths associated with dental scaling. Pak Oral Dental J.2016;36(2):267-9.
Cite this article as:

Dagar J. Rahar A, Gautam N, Dheeraj M. Dental Myths and Taboos: Hurdles to Oral Health. Int Healthc Res J. 2019;3(5):176-178. https://doi.org/10.26440/IHRJ/0305.08276

\section{Source of support: Nil, Conflict of interest: None}

\section{AUTHOR AFFILIATIONS AND CORRESPONDING ADDRESS:}

1. BDS ,MDS (Conservative Dentistry and Endodontics), International Dental and Cosmetic Centre, Gurugram, Haryana (Corresponding Author)

2. PG Student, Department of Conservative Dentistry and Endodontics, BRS Dental college and Hospital Sultanpur, Barwala, Panchkula Haryana

3. PG Student, Department of Pedodontics and Preventive Dentistry, BRS Dental college and Hospital Sultanpur, Barwala, Panchkula Haryana

4. BDS, MDS (Conservative Dentistry and Endodontics), Clinic Dental Hub, Private Practitioner, Jammu, Jammu and Kashmir 